\title{
Prediction of marker genes associated with hypertension by bioinformatics analyses
}

\author{
YUAN GAO, GUO-XIAN QI, ZHI-MEI JIA and YING-XIAN SUN \\ Department of Cardiology, The First Hospital of China Medical University, Shenyang, Liaoning 110001, P.R. China
}

Received December 22, 2015; Accepted May 9, 2017

DOI: 10.3892/ijmm.2017.3000

\begin{abstract}
This study aimed to explore the underlying marker genes associated with hypertension by bioinformatics analyses. A gene expression profile (GSE54015) was downloaded. The differentially expressed genes (DEGs) between the normotensive female (NF) and hypertensive female (HF), and between the normotensive male (NM) and hypertensive male (HM) groups were analyzed. Gene Ontology (GO) and pathway enrichment analyses were performed, followed by protein-protein interaction (PPI) network construction. The transcription factors (TFs), and the common DEGs between the HF and HM groups were then analyzed. In total, 411 DEGs were identified between the HF and NF groups, and 418 DEGs were identified between the HM and NM groups. The upregulated DEGs in the HF and HM groups were enriched in $9 \mathrm{GO}$ terms, including oxidation reduction, such as cytochrome $\mathrm{P} 450$, family 4 , subfamily $\mathrm{b}$, polypeptide 1 (Cyp4b1) and cytochrome P450, family 4, subfamily a, polypeptide 31 Cyp $4 a 31$ ). The downregulated DEGs were mainly enriched in GO terms related to hormone metabolic processes. In the PPI network, cytochrome P450, family 2, subfamily e, polypeptide 1 (Cyp2el) had the highest degree in all 3 analysis methods in the HF group. Additionally, 4 TFs were indentified from the 2 groups of data, including sterol regulatory element binding transcription factor 1 (Srebf1), estrogen receptor 1 (Esr1), retinoid X receptor gamma (Rxrg) and peroxisome proliferator-activated receptor gamma (Pparg). The intersection genes were mainly enriched in GO terms related to the extracellular region. On the whole, our data indicate that the DEGs, Cyp4b1, Cyp4a31 and Loxl2, and the TFs, Esrl, Pparg and Rxrg, are associated with the progression of hypertension, and may thus serve as potential therapeutic targets in this disease.
\end{abstract}

Correspondence to: Dr Yuan Gao, Department of Cardiology, The First Hospital of China Medical University, 155 Nanjing Street, Shenyang, Liaoning 110001, P.R. China

E-mail: plateau1216@163.com

Key words: hypertension, differentially expressed genes, proteinprotein interaction network, transcription factor

\section{Introduction}

Hypertension, also known as arterial hypertension, is a chronic medical condition in which the blood pressure in the arteries is elevated (1). Hypertension is classified as either primary or secondary hypertension, and primary hypertension accounts for approximately $90-95 \%$ of cases (2). In primary hypertension, the cause of the hypertensioin is not clearly defined, whereas in secondary hyptertension, the cause of the high blood pressure is clearly determined. Hypertension can lead to cardiac hypertrophy, aortic dissection and heart failure, due to structural changes in blood vessel walls and in the heart muscle, which has become a major burden to healthcare systems (3). Thus, hypertension is the leading risk factor for cardiovascular disease (4). According to a report of the World Health Organization in 2011, cardiovascular diseases are the leading cause of morbidity and mortality worldwide (5).

As a multifactorial disease, hypertension results from a complex interaction of environmental factors and genetic factors (6). Numerous common genetic variants, as well as some rare genetic variants have been identified to affact blood pressure $(7,8)$. For instance, bone morphogenetic protein receptor type 2 , which belongs to the transforming growth factor (TGF) $\beta$ superfamily of receptors, has been found to be mutated in the majority of cases of hypertension (9). Interleukin (IL)-17 has also been thought to be involved in hypertension due to its role in increasing the production of some other immune system chemical signals, such as IL-1, IL-6 and tumor necrosis factor (TNF)- $\alpha$ (10). Additionally, Austin et al (11) suggested that mutations in caveolin-1 are associated with pulmonary arterial hypertension. Despite advances in understanding the etiology of hypertension, the genetic basis responsible for this disease remains poorly elucidated.

Presently, there are many species of animals used as models of hypertension. In our study, the microarray dataset, GSE54015, which contained data from Schlager BPH/2J and BPN/3J mouse models was downloaded for analysis. of note, Puig et al (12) also used the same mouse models to identify potential pharmacological targets of hypertension. In this study, we identified the differentially expressed genes (DEGs) between normotensive mice and hypertensive mice of both genders. Additionally, we performed functional enrichment analyses and protein-protein interaction (PPI) networks analysis to study and identify the pathways and DEGs associated with hypertension. Furthermore, transcription factors (TFs) 
and DEGs in both hypertensive female (HF) and male (HM) groups were analyzed. The results of this study may aid in the diagnosis and treatment of hypertension.

\section{Data collection methods}

Affymetrix microarray data. The microarray data of GSE54015 were downloaded, which was deposited in the National Center of Biotechnology Information (NCBI) Gene Expression Omnibus (GEO) (http://www.ncbi.nlm.nih.gov/geo/) database by Chiu et al (2). The platform is [MoGene-1_1-st] Affymetrix Mouse Gene 1.1 ST array [transcript (gene) version]. In the study by Chiu et al (2), 12- to 13-week-old Schlager hypertensive male and hypertensive female $\mathrm{BPH} / 2 \mathrm{~J}$, and normotensive male and normotensive female BPN/3J mice ( $\mathrm{n}=6$ /group) were used, and their kidney tissues were collected for the microarrays. In the present study, 4 types of mouse samples were used for analysis: normotensive female (NF) BPN/3J, hypertensive female (HF) $\mathrm{BPH} / 2 \mathrm{~J}$, normotensive male (NM) BPN/3J and hypertensive male (HM) $\mathrm{BPH} / 2 \mathrm{~J}$ mice.

Data pre-processing and analysis of DEGs. The CEL raw data were downloaded and preprocessed using oligo (13) in $\mathrm{R}$ of Bioconductor (http://www.bioconductor.org/packages/ release $/ \mathrm{bioc} / \mathrm{html} /$ ) with the method of robust multiarray average (RMA) (14). The preprocessing processes included background correction, quartile data normalization and probe summarization.

The paired t-test based on the limma package (15) (no. 1406, Ritchie, 2015) in Bioconductor was used to identify the DEGs between the HF and NF groups, and between the HM and NM groups. The false discovery rate (FDR) was calculated to adjust the P-value with the Benjamini-Hochberg method (16). Only genes with $l \log _{2} \mathrm{FCl} \geq 0.58$ and an adjusted P-value $<0.05$ were regarded as DEGs.

Gene ontology (GO) and pathway enrichment analyses. The GO (http://www.geneontology.org) project is used to provide consistent descriptors for gene products in different databases, and to standardize classifications for sequences (17). Kyoto Encyclopedia of Genes and Genomes (KEGG) (http://www. genome.ad.jp/kegg/) is a knowledge base used to analyze gene functions, and to link genomic information with higher order functional information (18). In this study, the obtained DEGs were performed GO and KEGG pathway enrichment analyses to obtain the biological process (BP), molecular function (MF), cellular component (CC) terms and pathways. The analysis tool was the Database for Annotation, Visualization and Integrated Discovery (DAVID) (http://david.niaid.nih. gov) (19), which is an integrated biological knowledgebase used for the systematic and integrative analysis of large gene protein lists. The $P$-value $<0.05$ and enrichment count $\geq 2$ were considered as thresholds.

PPI network construction. The Search Tool for the Retrieval of Interacting Genes (STRING) (http://string-db.org/) database (20) is a precomputed global resource used for evaluating PPI information. In the present study, the STRING online tool was applied to predict the PPI pairs of DEGs with a combined score of $>0.4$. The PPI network was constructed using cytoscape (21). Additionally, 3 methods including degree centrality, betweenness centrality and subgraph centrality were used to analyze the hub gene in the PPI network using the CytoNCA cytoscape plugin (http://apps.cytoscape. org/apps/cytonca) (22).

TF analysis. In order to predict the role of TFs in hypertension, we selected the TFs from the DEG corresponding proteins, and predicted the target associations between the TFs and the DEGs based on the TRANSFAC database in the iRegulon cytoscape plugin (http://iregulon.aertslab.org) (23), as previously described. FDR on motif similarity $<0.05$ was regarded as the threshold.

Analysis of DEGs in the HF and HM groups. In the present study, we screened the common DEGs between the HF and HM groups in order to analyze the similarity of hypertension in females and males. Subsequently, these selected DEGs were subjected to GO functional enrichment analysis using DAVID. Furthermore, the enriched GO terms were subjected to correlation analysis using the enrichmentmap cytoscape plugin (http:// baderlab.org/Software/EnrichmentMap/) (24) (P<0.05).

\section{Results}

Identification of DEGs. In total, 201 upregulated and 210 downregulated DEGs were identified between the HF and NF groups. Moreover, 204 upregulated and 214 downregulated DEGs were identified between the HM and NM groups. The results are presented in the heatmaps in Fig. 1A and B.

GO and pathway enrichment analyses of DEGs. The upregulated DEGs in the HF group were mainly enriched in GO terms related to ion transport and oxidation reduction, and in the pathway of histidine metabolism. The downregulated DEGs were mainly enriched in the GO terms related to steroid metabolic process and microsome, and in the pathway of arachidonic acid metabolism (Table I).

In the HM group, the upregulated DEGs were mainly enriched in GO terms related to co-factor metabolic process and oxidation reduction, and in the pathway of PPAR signaling. The downregulated DEGs were mainly enriched in GO terms related to hormone metabolic process and endoplasmic reticulum (Table II).

PPI network construction. The constructed PPI network based on the DEGs between the HF and NF groups is shown in Fig. 2A. The PPI network consisted of 193 nodes and 301 interaction pairs. The top 10 hub genes were selected based on the analysis of degree centrality, betweenness centrality and subgraph centrality. Among the 10 genes, cytochrome P450, family 2, subfamily E, polypeptide 1 (Cyp2el) had the highest degree in all the 3 analysis methods (Table III).

The PPI network constructed by the DEGs between HM and NM included 182 nodes and 248 PPI interaction pairs (Fig. 2B). The top 10 hub genes respectively analyzed by 3 methods are shown in Table IV, namle UDP glucuronosyltransferase 2 family, polypeptide B37 (Ugt2b37), formimidoyltransferase cyclodeaminase $(F t c d)$ and cytochrome P450, family 4, subfamily a, polypeptide 14 (Cyp4a14). 
Table I. Functional enrichment analysis for the DEGs of the HF group.

\begin{tabular}{|c|c|c|c|c|}
\hline & & Term & Count & P-value \\
\hline \multirow[t]{11}{*}{ Up } & \multirow[t]{3}{*}{$\mathrm{BP}$} & GO:0006811-ion transport & 19 & $5.68 \mathrm{E}-05$ \\
\hline & & GO:0019751-polyol metabolic process & 4 & $6.56 \mathrm{E}-03$ \\
\hline & & GO:0055114-oxidation reduction & 13 & $1.66 \mathrm{E}-02$ \\
\hline & \multirow[t]{3}{*}{$\mathrm{CC}$} & GO:0005576-extracellular region & 32 & 4.98E-04 \\
\hline & & GO:0042383-sarcolemma & 4 & $1.17 \mathrm{E}-02$ \\
\hline & & GO:0005886-plasma membrane & 41 & $1.75 \mathrm{E}-02$ \\
\hline & \multirow[t]{3}{*}{ MF } & GO:0022836-gated channel activity & 10 & $1.00 \mathrm{E}-03$ \\
\hline & & GO:0005216-ion channel activity & 10 & $4.35 \mathrm{E}-03$ \\
\hline & & GO:0022838-substrate specific channel activity & 10 & $5.32 \mathrm{E}-03$ \\
\hline & \multirow[t]{2}{*}{ KEGG } & mmu00340:Histidine metabolism & 3 & $1.96 \mathrm{E}-02$ \\
\hline & & mmu03320:PPAR signaling pathway & 4 & $3.10 \mathrm{E}-02$ \\
\hline \multirow[t]{13}{*}{ Down } & \multirow[t]{3}{*}{$\mathrm{BP}$} & GO:0008202-steroid metabolic process & 9 & 7.92E-05 \\
\hline & & GO:0055114-oxidation reduction & 17 & $2.25 \mathrm{E}-04$ \\
\hline & & GO:0010565-regulation of cellular ketone metabolic process & 4 & $9.96 \mathrm{E}-04$ \\
\hline & \multirow[t]{3}{*}{$\mathrm{CC}$} & GO:0005792-microsome & 9 & $2.16 \mathrm{E}-04$ \\
\hline & & GO:0042598-vesicular fraction & 9 & 2.71E-04 \\
\hline & & GO:0005783-endoplasmic reticulum & 19 & $6.14 \mathrm{E}-04$ \\
\hline & \multirow[t]{3}{*}{ MF } & GO:0030414-peptidase inhibitor activity & 11 & $2.89 \mathrm{E}-06$ \\
\hline & & GO:0004866-endopeptidase inhibitor activity & 10 & $1.06 \mathrm{E}-05$ \\
\hline & & GO:0004857-enzyme inhibitor activity & 11 & $4.83 \mathrm{E}-05$ \\
\hline & \multirow[t]{4}{*}{ KEGG } & mmu00590:Arachidonic acid metabolism & 5 & $3.56 \mathrm{E}-03$ \\
\hline & & mmu00511:Other glycan degradation & 3 & $6.44 \mathrm{E}-03$ \\
\hline & & mmu00830:Retinol metabolism & 4 & $1.49 \mathrm{E}-02$ \\
\hline & & mmu04610:Complement and coagulation cascades & 4 & $1.94 \mathrm{E}-02$ \\
\hline
\end{tabular}

DEGs, differentially expressed genes; HF, hypertensive female; up, upregulated DGEs; down, downregulated DEGs; BP, biological process; CC, cellular component; MF, molecular function; KEGG, Kyoto Encyclopedia of Genes and Genomes.
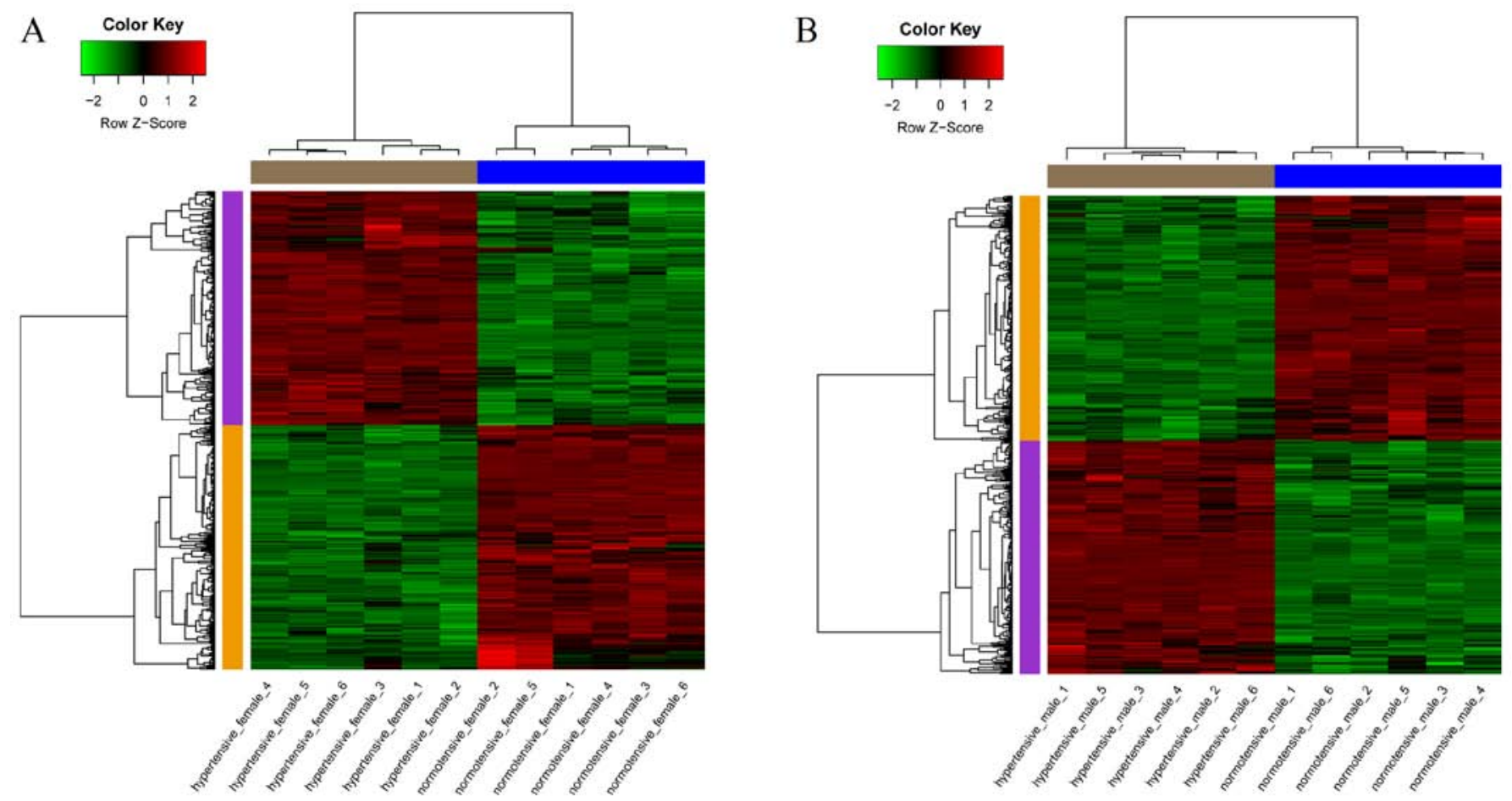

Figure 1. Heatmap plot of differentially expressed genes accross all samples. (A) Hypertensive female (HF) vs. normotensive female (NF); (B) hypertensive male (HM) vs. normotensive male (NM). Red stands for high expression value and green stands for low expression value. The changes of color from green to red stands for the changes of expression value from low to high. 
Table II. Functional enrichment analysis for the DEGs of the HM group.

\begin{tabular}{|c|c|c|c|c|}
\hline & & Term & Count & P-value \\
\hline \multirow[t]{11}{*}{ Up } & \multirow[t]{3}{*}{ BP } & GO:0051186-cofactor metabolic process & 8 & $1.04 \mathrm{E}-03$ \\
\hline & & GO:0055114-oxidation reduction & 15 & $2.04 \mathrm{E}-03$ \\
\hline & & GO:0019748-secondary metabolic process & 5 & $3.02 \mathrm{E}-03$ \\
\hline & \multirow[t]{3}{*}{$\mathrm{CC}$} & GO:0005576-extracellular region & 37 & $4.68 \mathrm{E}-06$ \\
\hline & & GO:0005777-peroxisome & 5 & $2.03 \mathrm{E}-02$ \\
\hline & & GO:0042579-microbody & 5 & 2.03E-02 \\
\hline & \multirow[t]{3}{*}{ MF } & GO:0019842-vitamin binding & 10 & $1.64 \mathrm{E}-06$ \\
\hline & & GO:0016290-palmitoyl-CoA hydrolase activity & 3 & $8.12 \mathrm{E}-03$ \\
\hline & & GO:0030170-pyridoxal phosphate binding & 4 & $1.33 \mathrm{E}-02$ \\
\hline & \multirow[t]{2}{*}{ KEGG } & mmu03320:PPAR signaling pathway & 5 & $6.25 \mathrm{E}-03$ \\
\hline & & mmu00590:Arachidonic acid metabolism & 5 & 7.43E-03 \\
\hline \multirow[t]{7}{*}{ Down } & \multirow[t]{3}{*}{ BP } & GO:0042445-hormone metabolic process & 7 & $1.63 \mathrm{E}-04$ \\
\hline & & GO:0010817-regulation of hormone levels & 7 & $1.07 \mathrm{E}-03$ \\
\hline & & GO:0042398-cellular amino acid derivative biosynthetic process & 4 & $9.31 \mathrm{E}-03$ \\
\hline & $\mathrm{CC}$ & GO:0005783-endoplasmic reticulum & 15 & $1.29 \mathrm{E}-02$ \\
\hline & \multirow[t]{3}{*}{ MF } & GO:0004866-endopeptidase inhibitor activity & 7 & $3.52 \mathrm{E}-03$ \\
\hline & & GO:0030414-peptidase inhibitor activity & 7 & $5.43 \mathrm{E}-03$ \\
\hline & & GO:0004867-serine-type endopeptidase inhibitor activity & 5 & 2.09E-02 \\
\hline
\end{tabular}

DEGs, differentially expressed genes; HM, hypertensive male; up, upregulated DGEs; down, downregulated DEGs; BP, biological process; CC, cellular component; MF, molecular function; KEGG, Kyoto Encyclopedia of Genes and Genomes.

Table III. The top 10 network nodes in the HF group based on the analysis of degree centrality, betweenness centrality and subgraph centrality.

\begin{tabular}{lclccc}
\hline Gene & Degree & & Betweenness & & Subgragh \\
\hline Cyp2e1 & 18 & Cyp2e1 & 7915.4395 & Cyp2e1 & 295.83835 \\
Cyp4a12a & 16 & Aldh1a1 & 4667.3003 & Cyp4a12a & 279.15607 \\
Cyp2b10 & 11 & Pla2g5 & 4572.402 & Cyp2b10 & 179.15228 \\
Pla2g5 & 9 & Srebf1 & 4370.598 & Slc22a30 & 100.235176 \\
Srebf1 & 9 & Dkk1 & 4108.5645 & Pla2g5 & 99.77931 \\
Serpinald & 9 & Cyp4a12a & 3427.9216 & Slco1a1 & 99.73588 \\
Cyp7b1 & 9 & Pld1 & 3171.1284 & Cyp4f18 & 81.0327 \\
Slc22a30 & 9 & Serpina1d & 3132.0713 & Cyp4f14 & 81.03269 \\
Anxa1 & 8 & Prok1 & 3062.5642 & Aldh1a1 & 73.60265 \\
Gm266 & 8 & Plc12 & 2823.1924 & Aldh1a7 & 69.66662 \\
\hline
\end{tabular}

HF, hypertensive female.

TF analysis. Based on the TRANSFAC database in iRegulon, the TFs in the HF and HM samples were predicted and the transcriptional regulatory networks were constructed (Fig. 3A and B). In the HF group, the TFs were sterol regulatory element binding transcription factor 1 (Srebf1) and estrogen receptor 1 (Esr1). In the HM group, the TFs were retinoid X receptor gamma (Rxrg) and peroxisome proliferator-activated receptor gamma (Pparg).

Analysis of DEGs in the HF and HM groups. There were 118 intersection genes among the upregulated DEGs in the
HF and HM samples; they were mainly distributed in chromosomes 4 and 7. Among the downregulated DEGs, 112 were intersection genes and they were mainly distributed in chromosomes 11 and 14. Moreover, these intersection genes were mainly enriched in BP terms related to catecholamine biosynthetic process, $\mathrm{CC}$ terms related to extracellular region and $\mathrm{MF}$ terms related to endopeptidase inhibitor activity (Table V). For instance, lysyl oxidase-like 2 (Loxl2) was enriched in the CC term related to extracellular region. The interaction network for these terms are shown in Fig. 4. 
A

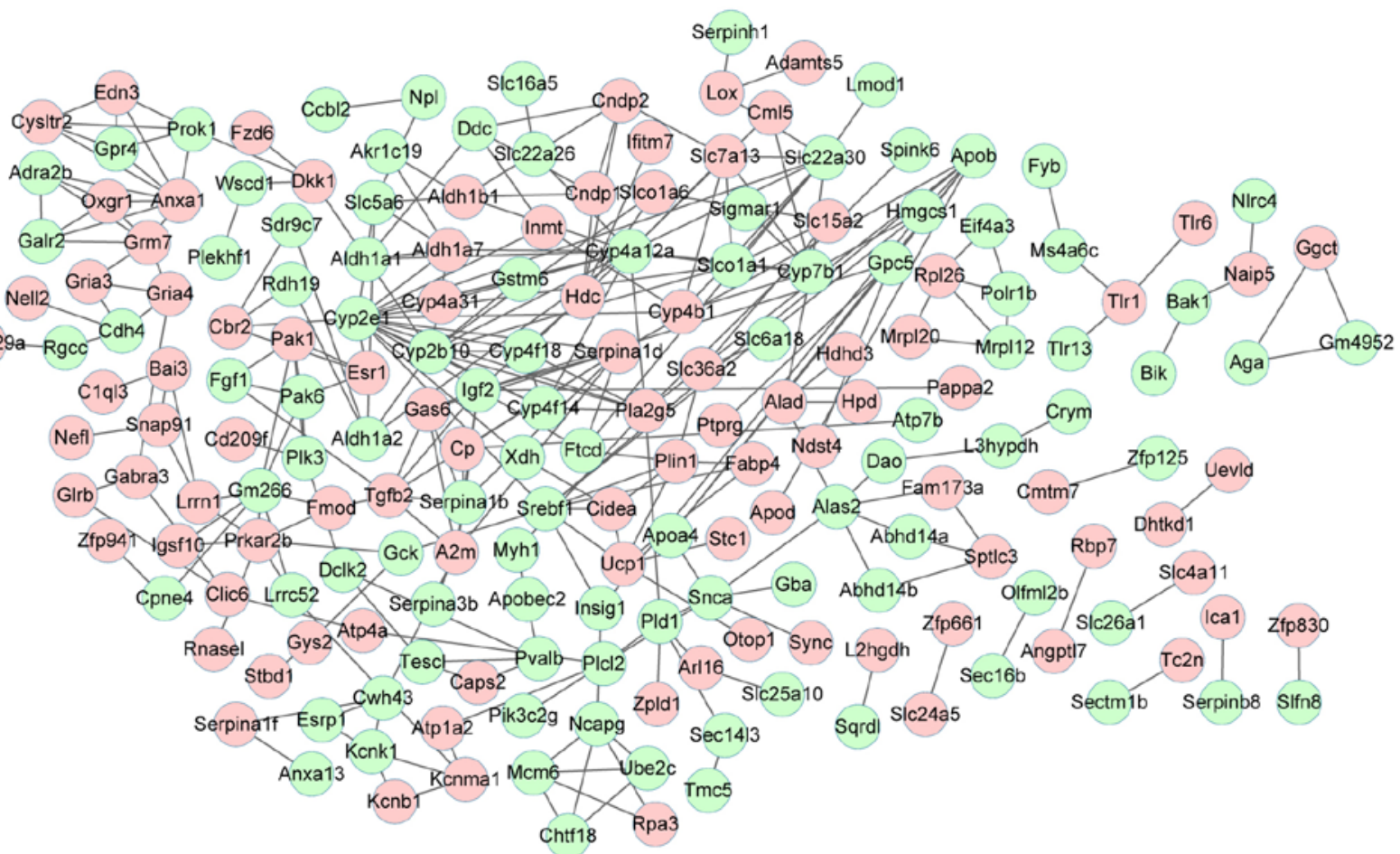

B
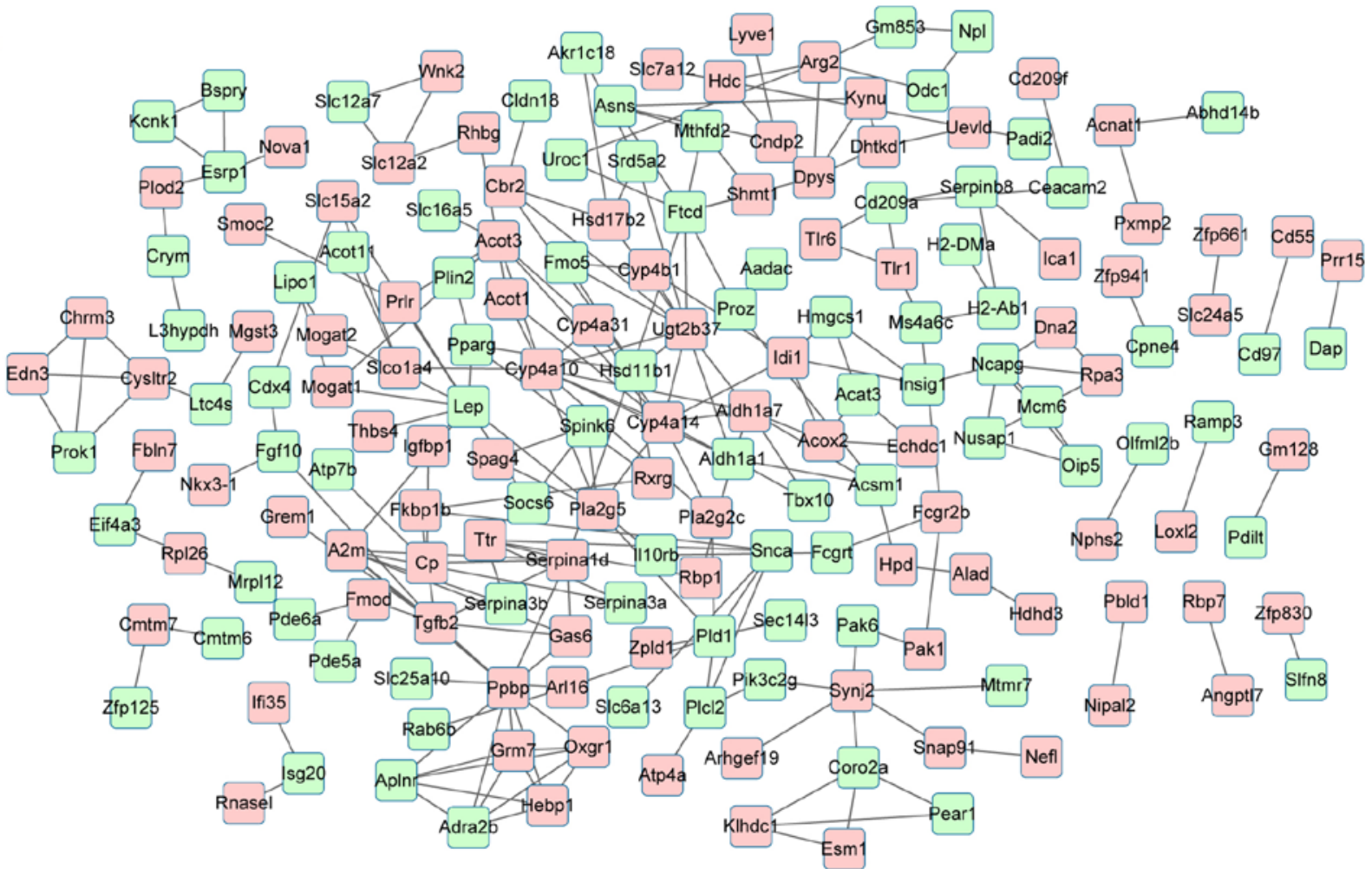

Figure 2. The constructed protein-protein interaction (PPI) network for differentially expressed genes (DEGs). Pink stands for upregulated DEGs and green stands for downregulated DEGs. (A) Hypertensive female (HF) vs. normotensive female (NF); (B) hypertensive male (HM) vs. normotensive male (NM).

\section{Discussion}

In the present study, 411 DEGs were identified between the HF and NF samples, and 418 DEGs were identified between the HM and NM samples. The upregulated DEGs, Cyp4bl and Cyp4a31, were mainly enriched in BP terms related to oxidation reduction in both the HF and HM samples. In addition, Esr1, Pparg and Rxrg were the key TFs involved in the transcriptional 
Table IV. The top 10 network nodes in the HM group based on the analysis of degree centrality, betweenness centrality and subgraph centrality.

\begin{tabular}{|c|c|c|c|c|c|}
\hline Gene & Degree & & Betweenness & & Subgragh \\
\hline Ugt2b37 & 11 & Ftcd & 5603.764 & Сур4а14 & 57.048008 \\
\hline Сур 4 al0 & 10 & Lep & 4363.4756 & Сур4а10 & 55.98465 \\
\hline Сур4а14 & 10 & Ugt2b37 & 4147.9277 & Ppbp & 52.882313 \\
\hline Lep & 10 & Serpina1d & 4005.5542 & Ugt2b37 & 50.838398 \\
\hline$P p b p$ & 9 & Cyp4a14 & 3500.8042 & Aldh1a7 & 34.876118 \\
\hline$T g f b 2$ & 8 & Fcgr $2 b$ & 2844.4468 & Aldh1a1 & 34.272568 \\
\hline Ftcd & 8 & Pld1 & 2784.8557 & Aplnr & 28.300505 \\
\hline Aldhla7 & 7 & Plcl2 & 2771.22 & Grm7 & 28.300499 \\
\hline Aldhlal & 7 & Il10rb & 2728.78 & Adra2b & 28.300493 \\
\hline$A 2 m$ & 7 & Synj2 & 2569.02 & Oxgr1 & 28.300491 \\
\hline
\end{tabular}

HM, hypertensive male.
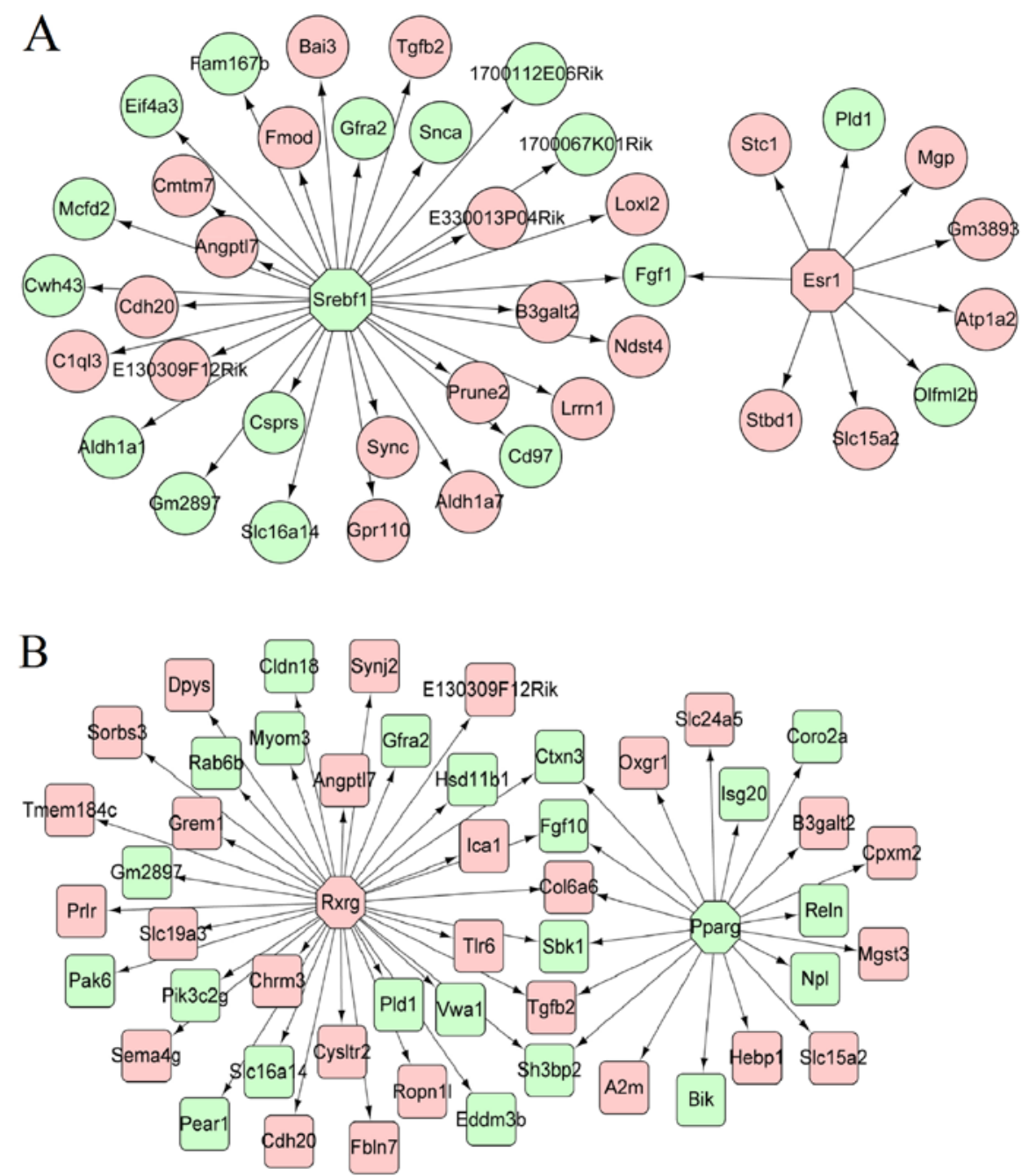

Figure 3. The constructed transcriptional regulatory network. Octagon stands for transcription factor; circle stands for differentially expressed genes (DEGs) in hypertensive female (HF); square stands for DEGs in hypertensive male (HM). Pink stands for upregulated DEGs and green stands for downregulated DEGs. (A) HF group; (B) HM group.

regulatory networks. Following the analysis of DEGs in the HF and HM samples, many intersection DEGs were identified between the 2 groups, such as Loxl2. These genes and TFs may play important roles in the progression of hypertension. 
Table V. The significant functions enriched by the intersection genes.

\begin{tabular}{|c|c|c|c|}
\hline & Term & Count & P-value \\
\hline \multirow[t]{3}{*}{ BP } & GO:0042423-catecholamine biosynthetic process & 3 & 0.004975549 \\
\hline & GO:0006638-neutral lipid metabolic process & 4 & 0.007615151 \\
\hline & GO:0042445-hormone metabolic process & 5 & 0.008732351 \\
\hline \multirow[t]{3}{*}{$\mathrm{CC}$} & GO:0005576-extracellular region & 31 & $4.56 \mathrm{E}-04$ \\
\hline & GO:0000267-cell fraction & 12 & 0.02798221 \\
\hline & GO:0005626-insoluble fraction & 11 & 0.030455631 \\
\hline \multirow[t]{3}{*}{ MF } & GO:0004866-endopeptidase inhibitor activity & 9 & $1.17 \mathrm{E}-04$ \\
\hline & GO:0030414-peptidase inhibitor activity & 9 & $2.16 \mathrm{E}-04$ \\
\hline & GO:0004867-serine-type endopeptidase inhibitor activity & 7 & $6.59 \mathrm{E}-04$ \\
\hline
\end{tabular}

$\mathrm{BP}$, biological process; CC, cellular component; MF, molecular function.

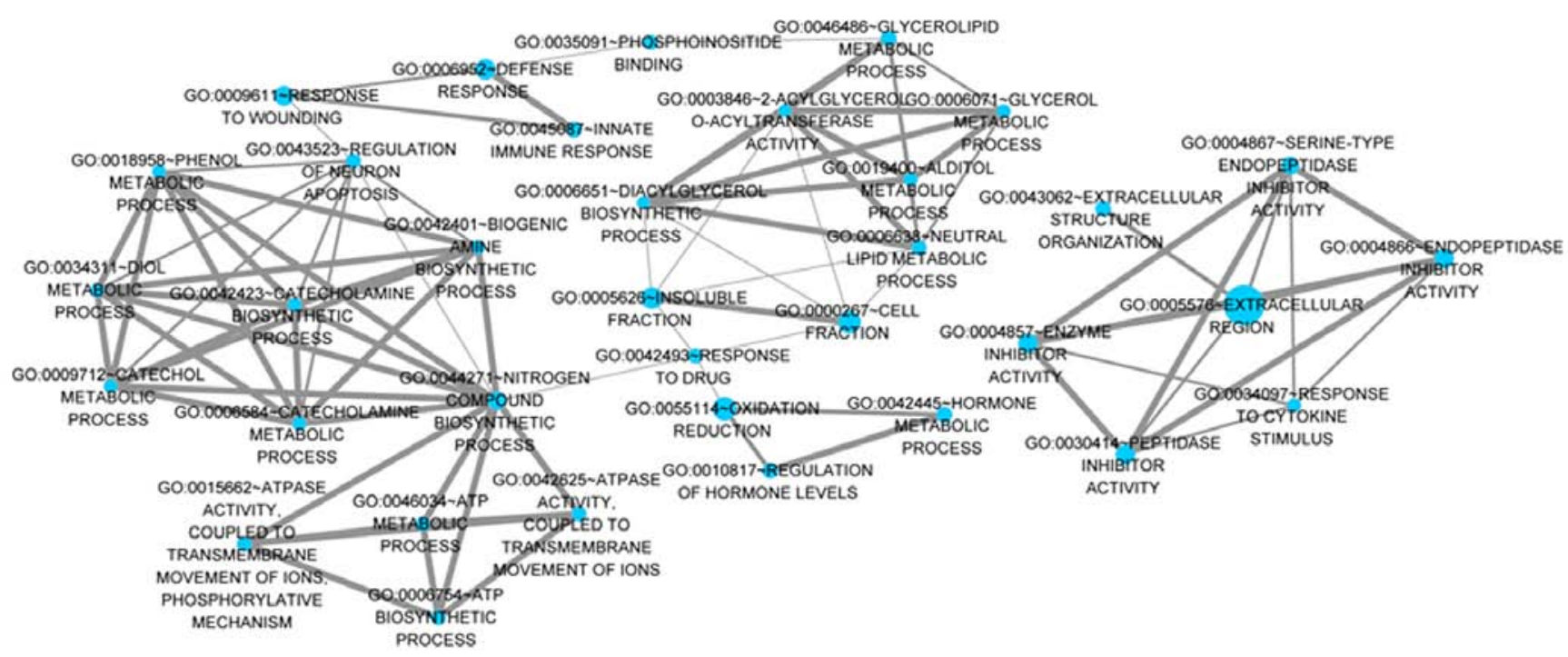

Figure 4. The interaction network for the terms enriched by intersection genes. Node stands for Gene Ontology (GO) term, the more the number of gene that was enriched in term, the bigger the node. Edge stands for the interaction between terms, the more the number of intersection genes, the thicker the edge.

Previous studies have indicated that reactive oxygen species play an important pathophysiological role in the development of cardiovascular diseases, including hypertension $(25,26)$. In human hypertension, biomarkers of systemic oxidative stress have been found to be elevated (27). In the present study, BP terms related to oxidation reduction were significant terms enriched by the DEGs in both the HF and HM samples. For instance, several cytochrome P450 (CYP)-associated genes, including Cyp $4 b 1$ and Cyp4a31 were enriched in this BP term. CYPs are important enzymes involved in the oxidative and reductive metabolism of numerous endogenous compounds in mammals (28). In the study by Chiu et al (2), Cyp4b1 was validated to be differentially expressed between hypertensive and normotensive mice by qPCR. CYP4A proteins have been found in vascular tissues (29) and have been suggested to be linked to hypertension in numerous experimental models (30). Sacerdoti et al (31) demonstrated that the inhibition or depletion of CYP4A activity can lower blood pressure in the spontaneously hypertensive rat. Of note, Cyp4a31 was found enriched in the PPAR signaling pathway in HF and HM samples. The PPARs are members of the nuclear receptor family of ligand-activated TFs, which play an important role in the regulation of cardiovascular circadian rhythms (32). Therefore, the upregulation of $C y p 4 b 1$ and $C y p 4 a 31$ in our study was in accordance with previous research. The 2 DEGs may be critical biomarkers associated with human hypertension.

In addition, Esr1 was a key TF in the transcriptional regulatory networks of the HF sample group. Esrl is a member of the family of ESRs, belonging to superfamily of nuclear receptor TFs. This receptor is expressed in a wide range of tissues and cells, including vascular smooth muscle and vascular endothelial cells (33). Estrogen can increase vasodilatation and inhibit the development of atherosclerosis (33). Specifically, the incidence of cardiovascular disease, including hypertension differs between males and females (34). Accumulating evidence suggests that estrogen deficiency plays a major role in the pathogenesis of cardiovascular diseases in post- 
menopausal women (35). In our study, the TF, Esr1, was also identified in female hypertensive mice, which indicated that Esr1 may play an important role in the progression of hypertension in females.

Furthermore, the TFs, Pparg and Rxrg, were involved in the transcriptional regulatory networks in the HM samples. Pparg is a member of PPARs which, as mentioned above, play an important role in the regulation of cardiovascular circadian rhythms. Pparg has been implicated in atherosclerosis (36), coronary heart disease (37) and hypertension (38). RXRs are members of the NR2B family of nuclear receptors and are major heterodimerization partners of PPARs (39). RXRs are involved in mediating the anti-proliferative effects of retinoic acid, which can comprise a collection of molecules with retinoids to maintain homeostasis in adults (40). A growing number of studies have reported the role of RXRs and PPARs in controlling cellular metabolism in tissues, including the myocardium (40,41). Nohara et al (39) suggested that a variant of Rxrg contributes to human genetic dyslipidemia. Taken together, the TFs, Pparg and Rxrg, may have utility in the diagnose and treatment of hypertension.

Following the analysis of DEGs in the HF and HM samples, we found that there were many intersection DEGs between the 2 groups, such as Loxl2. This DEG was found enriched in the $\mathrm{BP}$ terms related to oxidation reduction and $\mathrm{CC}$ terms related to extracellular region. Loxl2 encodes a member of the lysyl oxidase gene family, which are extracellular copper-dependent amine oxidases initiating the formation of crosslinks in collagen and elastin (42). The collagen and elastin are important components of the extracellular matrix (43). It has been found that the activity of lysyl oxidase is essential to maintain the features of connective tissues, including cardiovascular systems (44). The deregulation of lysyl oxidase is associated with aneurysms, aortic rupture and different stages of the atherosclerotic process $(45,46)$. Presently, there are few studies on the association between lysyl oxidase and hypertension. Thus, we speculated that Loxl2 may be a potential biomarker for the progression of hypertension.

In conclusion, our study provides a comprehensive bioinformatics analysis of biomarkers in hypertension, which may provide new insight into the mechanisms responsible for this disease. The DEGs, Cyp4b1, Cyp4a31 and Loxl2, and the TFs, Esrl, Pparg and Rxrg, may be associated with the progression of hypertension, and may serve as potential therapeutic targets in this disease.

However, there was a limitation to this study. The present study was implemented by means of bioinformatics; therefore, the screened genes and TFs have not been validated by actual experiments. As a result, further experimental studies with a larger sample size are warranted to confirm the results of our study.

\section{References}

1. Lupton SJ, Chiu CL and Lind JM: A hypertension gene: are we there yet? Twin Res Hum Genet 14: 295-304, 2011.

2. Chiu CL, Jackson KL, Hearn NL, Steiner N, Head GA and Lind JM: Identification of genes with altered expression in male and female Schlager hypertensive mice. BMC Med Genet 15: $101,2014$.

3. Williams SS: Advances in genetic hypertension. Curr Opin Pediatr 19: 192-198, 2007.
4. Chobanian AV, Bakris GL, Black HR, Cushman WC, Green LA, Izzo JL Jr, Jones DW, Materson BJ, Oparil S, Wright JT Jr, et al; Joint National Committee on Prevention, Detection, Evaluation, and Treatment of High Blood Pressure. National Heart, Lung, and Blood Institute; National High Blood Pressure Education Program Coordinating Committee: Seventh report of the joint national committee on prevention, detection, evaluation, and treatment of high blood pressure. Hypertension 42: 1206-1252, 2003.

5. Mendis S, Puska P and Norrving B (eds): Global atlas on cardiovascular disease prevention and control. World Health Organization, Geneva 2011.

6. Northcott CA, Glenn JP, Shade RE, Kammerer CM, Hinojosa-Laborde C, Fink GD, Haywood JR and Cox LA: A custom rat and baboon hypertension gene array to compare experimental models. Exp Biol Med (Maywood) 237: 99-110, 2012.

7. Ehret GB, Munroe PB, Rice KM, Bochud M, Johnson AD, Chasman DI, Smith AV, Tobin MD, Verwoert GC, Hwang SJ, et al; International Consortium for Blood Pressure Genome-Wide Association Studies; CARDIoGRAM consortium; CKDGen Consortium; KidneyGen Consortium; EchoGen consortium; CHARGE-HF consortium: Genetic variants in novel pathways influence blood pressure and cardiovascular disease risk. Nature 478: 103-109, 2011.

8. Lifton RP, Gharavi AG and Geller DS: Molecular mechanisms of human hypertension. Cell 104: 545-556, 2001.

9. Machado RD, Eickelberg O, Elliott CG, Geraci MW, Hanaoka M, Loyd JE, Newman JH, Phillips JA III, Soubrier F, Trembath RC, et al: Genetics and genomics of pulmonary arterial hypertension. J Am Coll Cardiol 54 (Suppl 1): S32-S42, 2009.

10. Gooch JL and Sharma AC: Targeting the immune system to treat hypertension: where are we? Curr Opin Nephrol Hypertens 23: 473-479, 2014.

11. Austin ED, Ma L, LeDuc C, Berman Rosenzweig E, Borczuk A, Phillips JA III, Palomero T, Sumazin P, Kim HR, Talati MH, et al: Whole exome sequencing to identify a novel gene (caveolin-1) associated with human pulmonary arterial hypertension. Circ Cardiovasc Genet 5: 336-343, 2012.

12. Puig O, Wang I-M, Cheng P, Zhou P, Roy S, Cully D, Peters M, Benita Y, Thompson J and Cai TQ: Transcriptome profiling and network analysis of genetically hypertensive mice identifies potential pharmacological targets of hypertension. Physiol Genomics 42A: 24-32, 2010.

13. Carvalho BS and Irizarry RA: A framework for oligonucleotide microarray preprocessing. Bioinformatics 26: 2363-2367, 2010.

14. Irizarry RA, Hobbs B, Collin F, Beazer-Barclay YD, Antonellis KJ, Scherf U and Speed TP: Exploration, normalization, and summaries of high density oligonucleotide array probe level data. Biostatistics 4: 249-264, 2003.

15. Smyth GK: Limma: linear models for microarray data. In: Bioinformatics and Computational Biology Solutions using $\mathrm{R}$ and Bioconductor. Gentleman R, Carey V, Huber W, Irizarry R and Dudoit S (eds). Springer, New York, pp397-420, 2005.

16. Ferreira JA: The Benjamini-Hochberg method in the case of discrete test statistics. Int J Biostat 3: 11, 2007.

17. Harris MA, Clark J, Ireland A, Lomax J, Ashburner M, Foulger R, Eilbeck K, Lewis S, Marshall B, Mungall C, et al; Gene Ontology Consortium: The Gene Ontology (GO) database and informatics resource. Nucleic Acids Res 32: D258-D261, 2004.

18. Kanehisa M and Goto S: KEGG: Kyoto encyclopedia of genes and genomes. Nucleic Acids Res 28: 27-30, 2000.

19. Huang W, Sherman BT and Lempicki RA: Systematic and integrative analysis of large gene lists using DAVID bioinformatics resources. Nat Protoc 4: 44-57, 2009.

20. Szklarczyk D, Franceschini A, Wyder S, Forslund K, Heller D, Huerta-Cepas J, Simonovic M, Roth A, Santos A, Tsafou KP, et al: STRING v10: protein-protein interaction networks, integrated over the tree of life. Nucleic Acids Res 43: D447-D452, 2015.

21. Shannon P, Markiel A, Ozier O, Baliga NS, Wang JT, Ramage D, Amin N, Schwikowski B and Ideker T: Cytoscape: a software environment for integrated models of biomolecular interaction networks. Genome Res 13: 2498-2504, 2003.

22. Tang Y, Li M, Wang J, Pan Y and Wu FX: CytoNCA: a cytoscape plugin for centrality analysis and evaluation of protein interaction networks. Biosystems 127: 67-72, 2015.

23. Janky R, Verfaillie A, Imrichová H, Van de Sande B, Standaert L, Christiaens V, Hulselmans G, Herten K, Naval Sanchez M, Potier D, et al: iRegulon: from a gene list to a gene regulatory network using large motif and track collections. PLoS Comput Biol 10: e1003731, 2014 
24. Merico D, Isserlin R, Stueker O, Emili A and Bader GD: Enrichment map: a network-based method for gene-set enrichment visualization and interpretation. PLoS One 5: e13984, 2010.

25. Touyz RM: Reactive oxygen species in vascular biology: role in arterial hypertension. Expert Rev Cardiovasc Ther 1: 91-106, 2003.

26. Chabrashvili T, Tojo A, Onozato ML, Kitiyakara C, Quinn MT, Fujita T, Welch WJ and Wilcox CS: Expression and cellular localization of classic NADPH oxidase subunits in the spontaneously hypertensive rat kidney. Hypertension 39: 269-274, 2002.

27. Redón J, Oliva MR, Tormos C, Giner V, Chaves J, Iradi A and Sáez GT: Antioxidant activities and oxidative stress byproducts in human hypertension. Hypertension 41: 1096-1101, 2003.

28. Nelson DR and Nebert DW: Cytochrome P450 (CYP) gene superfamily. eLS, 2011.

29. Marji JS, Wang M-H and Laniado-Schwartzman M: Cytochrome $\mathrm{P}-4504 \mathrm{~A}$ isoform expression and 20-HETE synthesis in renal preglomerular arteries. Am J Physiol Renal Physiol 283: F60-F67, 2002.

30. Singh H, Cheng J, Deng H, Kemp R, Ishizuka T, Nasjletti A and Schwartzman ML: Vascular cytochrome P450 4A expression and 20-hydroxyeicosatetraenoic acid synthesis contribute to endothelial dysfunction in androgen-induced hypertension. Hypertension 50: 123-129, 2007.

31. Sacerdoti D, Escalante B, Abraham NG, Mcgiff JC, Levere RD and Schwartzman ML: Treatment with tin prevents the development of hypertension in spontaneously hypertensive rats. Science 243: 388-390, 1989.

32. Kobayashi N, Ohno T, Yoshida K, Fukushima H, Mamada Y, Nomura M, Hirata H, Machida Y, Shinoda M, Suzuki N, et al: Cardioprotective mechanism of telmisartan via PPAR- $\gamma$-eNOS pathway in dahl salt-sensitive hypertensive rats. Am J Hypertens 21: 576-581, 2008.

33. Mendelsohn ME and Karas RH: The protective effects of estrogen on the cardiovascular system. N Engl J Med 340: 1801-1811, 1999.

34. Barrett-Connor E: Sex differences in coronary heart disease. Why are women so superior? The 1995 Ancel Keys Lecture. Circulation 95: 252-264, 1997.

35. Mendelsohn ME: Protective effects of estrogen on the cardiovascular system. Am J Cardiol 89: 12E-17E, 2002.

36. Kersten S, Desvergne B and Wahli W: Roles of PPARs in health and disease. Nature 405: 421-424, 2000.
37. Yilmaz-Aydogan H, Kurnaz O, Kurt O, Akadam-Teker B Kucukhuseyin O, Tekeli A and Isbir T: Effects of the PPARG P12A and C161T gene variants on serum lipids in coronary heart disease patients with and without type 2 diabetes. Mol Cell Biochem 358: 355-363, 2011.

38. Matsuda Y, Hoshikawa Y, Ameshima S, Suzuki S, Okada Y, Tabata T, Sugawara T, Matsumura Y, and Kondo T: Effects of peroxisome proliferator-activated receptor gamma ligands on monocrotaline-induced pulmonary hypertension in rats. Nihon Kokyuki Gakkai Zasshi 43: 283-288, 2005 (In Japanese).

39. Nohara A, Kawashiri M-A, Claudel T, Mizuno M, Tsuchida M, Takata M, Katsuda S, Miwa K, Inazu A, Kuipers F, et al: High frequency of a retinoid $X$ receptor $\gamma$ gene variant in familial combined hyperlipidemia that associates with atherogenic dyslipidemia. Arterioscler Thromb Vasc Biol 27: 923-928, 2007.

40. Sucov HM, Dyson E, Gumeringer CL, Price J, Chien KR and Evans RM: RXR alpha mutant mice establish a genetic basis for vitamin A signaling in heart morphogenesis. Genes Dev 8: 1007-1018, 1994

41. Osorio JC, Stanley WC, Linke A, Castellari M, Diep QN, Panchal AR, Hintze TH, Lopaschuk GD and Recchia FA: Impaired myocardial fatty acid oxidation and reduced protein expression of retinoid $\mathrm{X}$ receptor- $\alpha$ in pacing-induced heart failure. Circulation 106: 606-612, 2002.

42. Rodríguez C, Martínez-González J, Raposo B, Alcudia JF, Guadall A and Badimon L: Regulation of lysyl oxidase in vascular cells: lysyl oxidase as a new player in cardiovascular diseases. Cardiovasc Res 79: 7-13, 2008.

43. Mäki JM, Räsänen J, Tikkanen H, Sormunen R, Mäkikallio K, Kivirikko KI and Soininen R: Inactivation of the lysyl oxidase gene Lox leads to aortic aneurysms, cardiovascular dysfunction, and perinatal death in mice. Circulation 106: 2503-2509, 2002.

44. Lucero HA and Kagan HM: Lysyl oxidase: an oxidative enzyme and effector of cell function. Cell Mol Life Sci 63: 2304-2316, 2006.

45. Sibon I, Sommer P, Lamaziere JM and Bonnet J: Lysyl oxidase deficiency: a new cause of human arterial dissection. Heart 91: e33-e33, 2005.

46. Nakashima Y and Sueishi K: Alteration of elastic architecture in the lathyritic rat aorta implies the pathogenesis of aortic dissecting aneurysm. Am J Pathol 140: 959-969, 1992. 